\section{Incitatifs et contraintes dans l'activité médicale}

\author{
T. Perneger,$^{a, b}$ E. Chamot, ${ }^{a}$ P. Chastonay, ${ }^{a, c}$ P. Chopard, ${ }^{b, f}$ \\ P. Garnerin, ${ }^{b, g}$ F. Herrmann, ${ }^{\text {, e }}$ P. Bovier ${ }^{b, d}$ \\ a Institut de médecine sociale et préventive, Université de Genève \\ b Unité qualité des soins, Hôpitaux Universitaires de Genève \\ c Unité de développement et de recherche en éducation médicale \\ d Département de médecine communautaire, \\ Hôpitaux Universitaires de Genève \\ e Hôpital de Gériartrie, HUG \\ f Département de médecine interne, HUG \\ g Division d'anesthésiologie, HUG
}

\begin{abstract}
Résumé
Ce texte aborde la pratique médicale dans son contexte social et économique. Les incitatifs et contraintes auxquels sont soumis les médecins et les hôpitaux sont passés en revue, ainsi que leurs conséquences pour la pratique clinique. Ensuite, les différences entre la pratique libérale de la médecine qui prévaut actuellement en Suisse et les organisations de type "HMO" sont examinées. En conclusion, la régulation du système de santé est une affaire complexe, dont les médecins se doivent de connaître au moins les rudiments.
\end{abstract}

\section{Le mythe de l'indépendance du médecin}

Certains considèrent que la pratique de la médecine suit grosso modo le modèle du marché libéral: le patient choisit librement son médecin (ou son hôpital), reçoit de sa part des services, et le rétribue; le médecin pratique son art sans contrainte; il ne se préoccupe que du patient qui l'a consulté. La réalité étant assez différente, le médecin doit comprendre les influences des autres acteurs et les contraintes sur son activité qui en découlent. Ces acteurs sont:

\section{Les patients}

Le contrat tacite entre patient et médecin implique que ce dernier fera son possible pour résoudre les problèmes du premier. Beaucoup de médecins reconnaissent la primauté de cette relation. Mais il peut ar-

Correspondance:

Dr Philippe Chastonay

Centre médical universitaire

Institut de médecine sociale et préventive

Rue Michel-Servet

CH-1211 Genève 4 river que les demandes d'un patient interfèrent avec celles d'un autre; ceci est vrai pour la disponibilité du médecin, et aussi parfois pour les ressources disponibles, lorsque le médecin travaille avec un budget limité.

\section{La société}

Elle offre au médecin certains avantages (formation subventionnée, clientèle solvable garantie, etc.), mais en retour, elle acquiert un droit de regard sur ses activités. La société délègue aussi au médecin certaines fonctions autres que les soins. Ainsi, le médecin attribue officiellement le label de "malade" au patient, ce qui confère une série de droits à ce dernier (ex: arrêt de travail, prestations d'assurance-invalidité); il peut restreindre la responsabilité légale d'un accusé, ou exempter une recrue du service militaire.

Le médecin joue aussi un rôle en santé publique. Il trace les contacts d'un patient atteint d'une maladie transmissible (méningite, etc.), déclare certaines maladies aux autorités pour permettre d'en suivre l'évolution dans la population, propose les vaccinations non seulement pour protéger le patient, mais aussi pour bloquer la transmission de la maladie par «immunité de groupe». Le médecin doit aussi faire avancer les connaissances médicales en participant à la recherche clinique.

\section{Les payeurs}

Le médecin qui accepte de traiter des patients couverts par l'assurance-maladie de base doit se conformer à la LAMal, qui l'oblige à tenir compte du caractère économique des traitements, de leur efficacité, et de leur adéquation (art. 32). Cette loi formule également des exigences dans le domaine de la qualité des soins (art. 58). Le médecin est tenu de transmettre certaines informations aux assureurs, dans les limites de la législation sur la protection des données.

\section{Lui-même}

On oublie parfois que le médecin est aussi responsable de son propre bien-être. Le médecin se préoccupera du temps consacré aux activités non-professionnelles, du prestige dérivé de sa position, de son autonomie, de sa satisfaction professionnelle, et bien entendu de son revenu.

A ce propos, l'éventualité que les médecins payés à l'acte puissent générer le revenu qu'ils souhaitent (demande induite) préoccupe les payeurs. En Suisse, la densité médicale de chaque canton corrèle avec les coûts des soins ambulatoires par habitant [1] (fig. 1).

Peut-on conclure pour autant que la demande induite est la cause de cette association? Pas vraiment, car plusieurs autres explications peuvent être avancées (p.ex. les médecins s'installent là où une demande justifiée existe). Néanmoins, la seule possibilité que le mode de rétribution du médecin influence son activité pose la question plus générale des circonstances, autres que l'intérêt du patient, qui influencent la pratique médicale. 


\section{Figure 1}

Densité médicale par 10000 habitants à rapports des coûts moyens des soins ambulatoires.

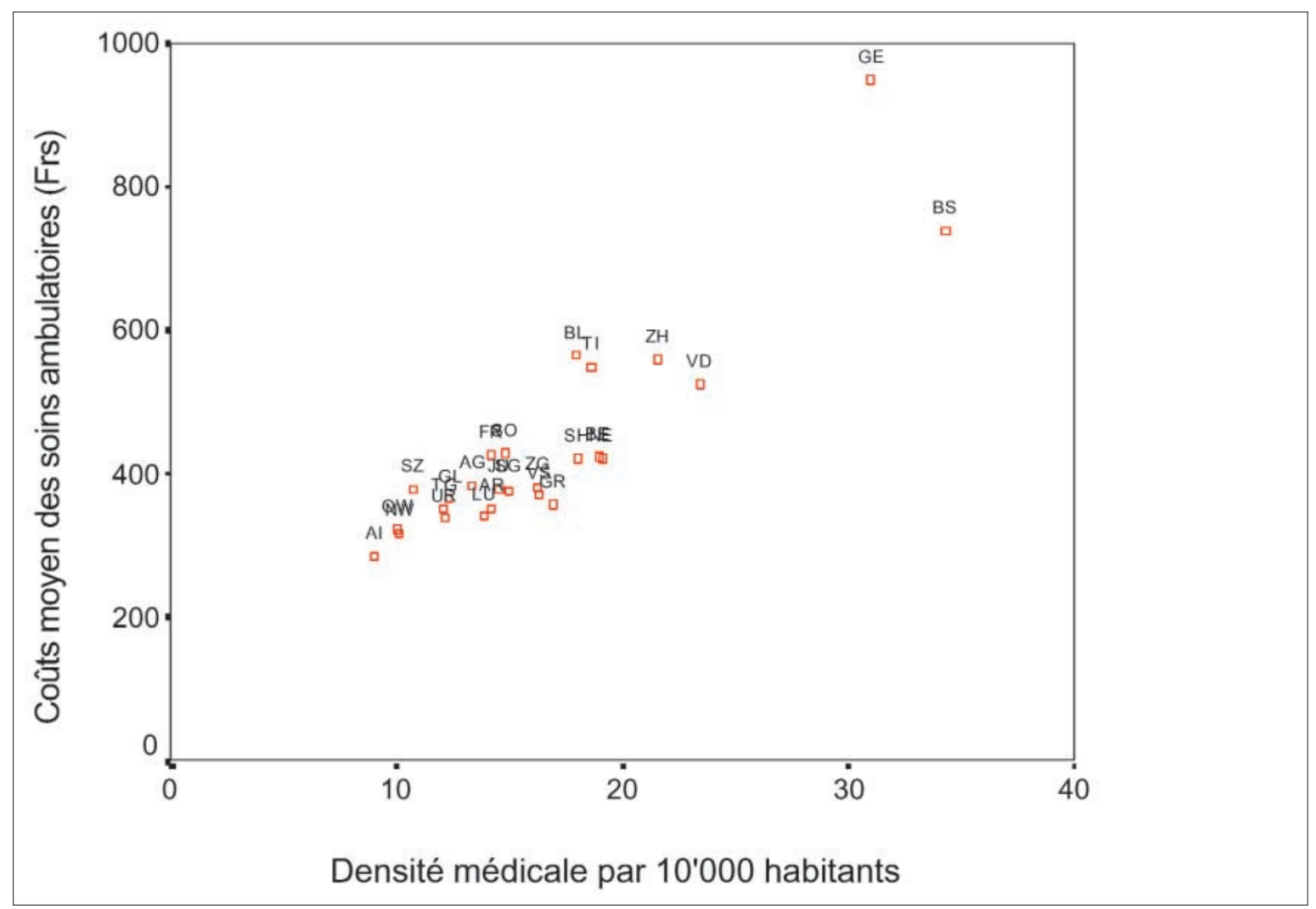

\section{Incitatifs et contraintes administratives}

Tout système de santé fonctionne avec des règles qui influencent les acteurs de ce système (patients, médecins, payeurs, ...), sans que ces derniers ne soient nécessairement conscients d'adapter leur comportement aux incitatifs et contraintes mis en place. Par exemple, le système de santé des Etats-Unis coûte trois fois plus cher que celui du Royaume-Uni, sans qu'on puisse affirmer que le Dr. $\mathrm{X}$ anglais prive son patient A d'un traitement utile, ou que le Dr. Y américain abuse d'examens inutiles chez son patient $B$.

Les incitatifs auxquels sont soumis les médecins sont de deux ordres: financiers et non-financiers. Le principal incitatif financier est le mode de rétribution (tab. 1). Aucun mode de paiement n'est idéal ou neutre; chacun influence la pratique du médecin ou de l'hôpital. Des systèmes de paiement mixtes, combinant par exemple les forfaits et le paiement à l'acte, peuvent aussi être appliqués. Certaines organisations utilisent encore des primes à la performance, liées à la satisfaction des patients ou à des indicateurs de qualité des soins.

Plusieurs effets prévisibles des méthodes de paiement citées ci-dessus ont été clairement démontrés: par exemple, des médecins d'une policlinique pédiatrique universitaire payés à l'acte ont programmé plus de visites chez leurs patients que les médecins qui sont restés salariés, et ceci surtout pour des patients en bonne santé [2].
Le mécanisme de remboursement influence non seulement les médecins à titre individuel, mais aussi les hôpitaux. Lorsqu'aux Etats-Unis on est passé d'un paiement à l'acte à un paiement forfaitaire par hospitalisation, la prise en charge de patients hospitalisés pour fracture de hanche a profondément changé [3]. La durée médiane de séjour est passée de 19 à 12 jours, la physiothérapie a débuté en moyenne 4 jours plus tôt, mais seulement $40 \%$ des patients pouvaient marcher lors de la sortie, par rapport à 56\% précédemment, et le périmètre moyen de marche à la sortie est passé de 27 mètres à 11 mètres. Les patients étaient donc pris en charge plus rapidement, mais ils sortaient aussi plus vite de l'hôpital, et moins complètement réhabilités. Le paiement forfaitaire favorise non seulement l'efficacité, mais aussi le transfert d'une partie des soins, et des coûts correspondants, vers d'autres établissements.

L'importance du mode de remboursement se manifeste aussi dans les débats actuels autour de l'introduction du nouveau tarif des prestations médicales en Suisse (TARMED). Jusqu'à présent, les tarifs avaient une base historique, sans justification explicite. Bien que ces tarifs varient entre cantons, ils favorisent généralement les gestes techniques, à tel point qu'on entend parfois dire que "sans mon laboratoire je ne tourne pas.» TARMED tente d'introduire une plus grande transparence, d'une part en séparant les frais logistiques (matériel, assistance, etc.) de l'acte purement médical, et d'autre part en introduisant une 
formule explicite pour le calcul de chaque coût de prestation: durée, difficulté, risque, formation nécessaire, etc. TARMED tente également de donner plus de poids à l'acte intellectuel, plutôt qu'à l'acte technique. Ceci est bien entendu une décision politique, dérivée d'une certaine vision de ce que doivent être les soins de qualité. De façon prévisible, cette proposition a causé une tension entre les médecins généralistes et les spécialistes techniques.

\section{Les organisations de type "managed care" ou "health maintenance organization"}

Il existe également des moyens non-financiers d'influencer l'activité médicale. Certains sont tellement évidents qu'on ne les perçoit pas comme tels: droit de pratique, nécessité d'une formation continue, code de déontologie, culture professionnelle du travail bien fait. Un moyen de contrôle plus évident est du ressort des assureurs, qui peuvent demander au médecin ou à l'hôpital de justifier certaines factures. Mais c'est dans les organisations de type "managed care» ou "health maintenance organization» (HMO) que des moyens plus contraignants sont utilisés. Ces moyens sont évoqués dans le tableau 2.

La différence fondamentale entre l'assurance maladie ordinaire et une organisation de type "managed care» (traduction: "soins gérés») est que ces dernières intègrent les fonctions d'assureur et de fournisseur de soins. Ces organisations doivent non seulement rembourser les frais médicaux, mais aussi les fournir, si possible à moindres frais que dans le système traditionnel. Ceci implique que des administrateurs doivent contrôler l'offre et la demande de soins, ce qui n'a pas cours dans un marché libéral. Ces organisations, qui se sont développées aux Etats-Unis, sont maintenant autorisées par la LAMal en Suisse.

Il y plusieurs sortes de HMO. Le système le plus ancien est le cabinet de groupe qui salarie ses médecins et autres soignants, et qui répartit les tâches des uns et des autres de façon coordonnée. Typiquement, les patients sont attribués à des médecins de premier recours désignés (qu'on appelle parfois "gatekeeper»). Ces médecins s'occupent des problèmes de santé courants et décident ou non d'envoyer le patient chez un spécialiste ou à l'hôpital, et d'approuver le cas échéant les traitements chers. Ceci est une HMO classique.

Le réseau de médecins indépendants est une organisation un peu plus lâche. Il en existe deux variétés de base. La première ressemble à une HMO décentralisée, qui repose sur des médecins de premier recours, qui exercent un contrôle assez serré sur l'accès aux spécialistes, et qui sont fréquemment payés par capitation (forfait par assuré par mois). Ces médecins peuvent travailler au sein d'un ou de plusieurs réseaux

\section{Tableau 1}

Modes de rétribution des médecins ou établissements de santé.

\begin{tabular}{|c|c|c|c|}
\hline Mode de paiement & Mécanisme & Avantages & Inconvénients \\
\hline \multirow[t]{3}{*}{ A l'acte } & \multirow[t]{3}{*}{ Chaque prestation est payée séparément } & Le médecin maîtrise son revenu & Surutilisation des prestations \\
\hline & & Autonomie & Coûts administratifs élevés \\
\hline & & Incitation à satisfaire le patient & Coûts totaux élevés \\
\hline A l'acte, avec une retenue & $\begin{array}{l}\text { Une partie (p.ex. } 10-20 \% \text { ) du paiement } \\
\text { est retenue et versée seulement } \\
\text { si l'exercice financier annuel le permet }\end{array}$ & $\begin{array}{l}\text { Incitation à économiser pour éviter } \\
\text { de perdre la retenue }\end{array}$ & $\begin{array}{l}\text { Augmentation du nombre d'actes, } \\
\text { pour "se rattraper" }\end{array}$ \\
\hline \multirow[t]{4}{*}{ Forfait annuel (capitation) } & $\begin{array}{l}\text { Chaque patient (ou assuré) affilié } \\
\text { au médecin rapporte un forfait fixe }\end{array}$ & Budget global fixé & $\begin{array}{l}\text { Le forfait doit tenir compte } \\
\text { de l'état de santé des patients }\end{array}$ \\
\hline & \multirow[t]{3}{*}{ Fonds d'assurance pour cas très chers } & $\begin{array}{l}\text { Aucune incitation à faire } \\
\text { des actes inutiles }\end{array}$ & Risque de rationnement \\
\hline & & Faible coûts administratifs & \\
\hline & & Maîtrise des coûts & \\
\hline \multirow[t]{2}{*}{ Salaire } & \multirow[t]{2}{*}{$\begin{array}{l}\text { Revenu mensuel fixe indépendant } \\
\text { de l'activité }\end{array}$} & $\begin{array}{l}\text { Neutre: pas d'incitation à en faire } \\
\text { trop ou pas assez }\end{array}$ & $\begin{array}{l}\text { Ne récompense pas l'initiative } \\
\text { ou la productivité individuelle }\end{array}$ \\
\hline & & Maîtrise des coûts & \\
\hline \multirow[t]{3}{*}{ Forfait par épisode de soins } & \multirow[t]{3}{*}{$\begin{array}{l}\text { Paiement forfaitaire par hospitalisation } \\
\text { (aussi envisageable en ambulatoire) }\end{array}$} & $\begin{array}{l}\text { Incitation à maîtriser le coût total } \\
\text { par épisode de soins }\end{array}$ & $\begin{array}{l}\text { Le forfait doit tenir compte } \\
\text { de l'état de santé des patients }\end{array}$ \\
\hline & & Maîtrise des coûts & $\begin{array}{l}\text { Manipulation du codage } \\
\text { pour maximiser le revenu }\end{array}$ \\
\hline & & & $\begin{array}{l}\text { Incite à écourter les séjours } \\
\text { ou à rationner }\end{array}$ \\
\hline \multirow[t]{2}{*}{ Forfait par journée } & \multirow{2}{*}{$\begin{array}{l}\text { Paiement forfaitaire par jour } \\
\text { d'hospitalisation }\end{array}$} & \multirow[t]{2}{*}{ Simplicité } & Incite à remplir tous les lits \\
\hline & & & $\begin{array}{l}\text { Décourage tentatives d'écourter } \\
\text { les séjours }\end{array}$ \\
\hline
\end{tabular}


Tableau 2

Mesures administratives visant à maîtriser les coûts de la santé.

\begin{tabular}{|c|c|c|}
\hline Mesure & Avantages & Inconvénients \\
\hline $\begin{array}{l}\text { Approbation par l'assureur des procédures } \\
\text { chères («utilization review») }\end{array}$ & Réduction d'une utilisation inadéquate & Perte d'autonomie des médecins \\
\hline \multirow{2}{*}{$\begin{array}{l}\text { Application systématique } \\
\text { de recommandations } \\
\text { pour la pratique clinique }\end{array}$} & Approche clinique cohérente & Perte d'autonomie des médecins (?) \\
\hline & $\begin{array}{l}\text { Permet d'intégrer l'uevidence-based medicine» } \\
\text { dans la pratique }\end{array}$ & $\begin{array}{l}\text { Difficultés à tenir à jour } \\
\text { les recommandations }\end{array}$ \\
\hline \multirow{2}{*}{$\begin{array}{l}\text { Exclusion des médecins } \\
\text { dont les coûts par cas sont élevés }\end{array}$} & Coûts bas au sein de l'organisation & Coûts élevés hors de l'organisation \\
\hline & & $\begin{array}{l}\text { Ne tient pas compte de l'état } \\
\text { de santé des patients }\end{array}$ \\
\hline
\end{tabular}

tout en gardant une clientèle privée. A l'autre extrême se trouvent des associations de médecins peu contraignantes, où les médecins sont payés à l'acte et restent librement accessibles aux assurés, mais octroient un rabais à l'assureur sur les tarifs usuels. Ces organisations-types ne sont décrites ici qu'à titre indicatif. Dans la réalité, toutes sortes de montages peuvent exister, qui associent à divers degrés des incitatifs financiers (tab. 1) et non-financiers (tab. 2).

Il convient aussi de connaître le statut de ces organisations. Aux Etats-Unis, certaines HMO sont des entreprises commerciales (comme les assurances complémentaires en Suisse), alors que d'autres n'ont pas de but lucratif (comme l'assurance-maladie de base). Une étude récente a montré que la qualité des soins, mesurée par des indicateurs tels que la proportion d'assurés immunisés conformément aux recommandations ou la proportion de diabétiques bénéficiant d'un examen ophtalmologique annuel, étaient moins bonne dans les HMO à but lucratif que dans les HMO sans but lucratif [4]. De plus, la proportion des revenus consacrée aux soins était de 81\% pour les premières, et $87 \%$ pour les secondes. Or, ce sont justement les HMO à but lucratif qui se développent le plus aux Etats-Unis. Ceci suggère que la recherche du profit privé peut nuire à la qualité des soins, et que les lois du marché ne favorisent pas nécessairement les organisations les plus efficaces.

Un médecin qui envisage d'adhérer à l'une de ces organisations doit décider si le mélange d'incitatifs et de mesures d'économie proposé est acceptable ou si au contraire ces mesures risquent de causer des conflits d'intérêt trop importants, lésant les intérêts des patients [5].

\section{Conclusion}

Le fonctionnement de tout système de santé est déterminé par le jeu des incitatifs et contraintes mis en place. Le médecin, acteur central du système, est une cible de choix (mais l'assuré également: songeons aux franchises et participations aux frais). Par définition, le médecin ne peut y échapper, puisque même les systèmes que l'on croit libéraux sont en fait régis par des lois, règlements, modes de remboursement, etc. Comme les systèmes de santé de la plupart des pays sont en mutation, en partie à cause de l'augmentation des coûts de la santé, le médecin doit comprendre comment un changement des règles du jeu va affecter ses relations avec les autres partenaires ou acteurs, et en priorité avec ses patients.

\section{Références}

1 Domenighetti G. Marché de la santé: Ignorance ou adéquation? Lausanne. Editions Réalités sociales; 1994.

2 Hickson GB, Altheimer WA, Perrin JM. Physician reimbursement by salary or fee-for-service: effect on physician practice behavior in a randomized prospective study. Pediatrics 1987;80:344-50.

3 Fitzgerald JF, Moore PS, Dittus RS. The care of elderly patients with hip fracture. Changes since implementation of the prospective payment system. N Engl J Med 1988; 319:1392-7.

4 Himmelstein DU, Woolhandler S, Hellander I, Wolfe SM. Quality of care in investor-owned vs not-for-profit HMOs. JAMA 1999;282:159-63.

5 Emanuel EJ, Dubler NN. Preserving the physician-patient relationship in the era of managed care. JAMA 1995; 273:323-9. 